\title{
Phase I Study of Yttrium-90 Radiolabeled M5A Anti-Carcinoembryonic Antigen Humanized Antibody in Patients with Advanced Carcinoembryonic Antigen Producing Malignancies
}

\author{
David Akhavan,, Paul Yazaki, ${ }^{1}$ Dave Yamauchi, ${ }^{3}$ Jennifer Simpson, ${ }^{4}$ Paul H. Frankel, ${ }^{5}$ James Bading, \\ David Colcher, ${ }^{2}$ Kofi Poku, ${ }^{2}$ Yi-jen Chen, ${ }^{1}$ Dean Lim, ${ }^{4}$ Mihaela Cristea, ${ }^{4}$ Anna Wu, \\ John Shively, ${ }^{2}$ and Jeffrey Y.C. Wong ${ }^{1}$
}

\begin{abstract}
Background: M5A is a humanized monoclonal antibody (mAb) directed against carcinoembryonic antigen (CEA) The purpose of this first in human phase I dose-escalation trial was to characterize the toxicities and determine the maximum tolerated dose (MTD) of yttrium-90 $\left({ }^{90} \mathrm{Y}\right)$-DOTA-M5A as a single agent and in combination with gemcitabine (gem).

Methods: Patients with advanced metastatic CEA-producing malignancies who had progressed on standard therapies were first administered indium-111 $\left({ }^{111}\right.$ In)-DOTA-M5A. If tumor targeting was observed, the patient then received the therapy dose of ${ }^{90}$ Y-DOTA-M5A. Serial scans, blood sampling, and $24 \mathrm{~h}$ urine collections were then performed to estimate radiation doses to organs and total body. Assays for human antihuman antibody (HAHA) responses were performed out to 6 months.

Results: Of the 18 patients who received ${ }^{111}$ In-DOTA-M5A, 16 received ${ }^{90}$ Y-DOTA-M5A therapy; 1 patient at 14 $\mathrm{mCi} / \mathrm{m}^{2}$ with gem $\left(150 \mathrm{mg} / \mathrm{m}^{2}\right.$ days 1 and 3$), 3$ patients at $12 \mathrm{mCi} / \mathrm{m}^{2}$ with gem, 6 patients at $12 \mathrm{mCi} / \mathrm{m}^{2}$ without gem, and 6 at $10 \mathrm{mCi} / \mathrm{m}^{2}$ without gem. Prolonged cytopenias resulted in discontinuation of dose escalation with gemcitabine. A single agent MTD of $10 \mathrm{mCi} / \mathrm{m}^{2}$ was established based on dose-limiting hematopoietic toxicities. HAHA immune response was identified in 2 of 16 patients (12.5\%). Stable disease at 3 months was seen in 10 patients and 2 patients demonstrated an $88 \%$ and $64 \%$ decrease in CEA back to normal levels. In 2 patients ${ }^{111}$ InDOTA-M5A imaging revealed previously unknown brain metastases.

Conclusion: This study demonstrates the potential utility of the ${ }^{90}$ Y-DOTA-M5A anti-CEA mAb as a therapeutic antibody. There is decreased immunogenicity compared with murine and chimeric mAbs, allowing for the potential of multiple administrations. Combined modality therapy approaches incorporating this agent should continue to be evaluated.
\end{abstract}

Keywords: carcinoembryonic antigen, radioimmunotherapy colorectal cancer, medullary thyroid cancer, yttrium-90

\section{Introduction}

$\mathbf{R}$ adioimmunotherapy (RIT), defined as the use of radiolabeled antibodies to specifically target therapeutic doses of radiation to tumor, offers a theoretically attractive delivery system for systemic-guided radiation therapy. Objective responses have been observed primarily in radiosensitive hematologic malignancies. ${ }^{1,2}$ Radiolabeled antibodies have been successful in targeting comparable radiation doses with solid tumors as lymphomas, with estimated tumor doses

${ }^{1}$ Department of Radiation Oncology, City of Hope National Cancer Center, Duarte, California.

${ }^{2}$ Department of Molecular Imaging and Therapy, Beckman Research Institute, Duarte, California.

${ }^{3}$ Department of Diagnostic Radiology, City of Hope National Cancer Center, Duarte, California.

${ }^{4}$ Department of Medical Oncology, City of Hope National Cancer Center, Duarte, California.

${ }^{5}$ Department of Information Sciences, City of Hope National Cancer Center, Duarte, California.

Address correspondence to: Jeffrey Wong; Department of Radiation Oncology, City of Hope National Cancer Center; 1500 E. Duarte 
capable of two to three log kill in solid tumors. However, objective responses have been limited when RIT is used as monotherapy due to the relative lack of radiosensitivity of these cancers. As a result trials have evaluated adding RIT to established single agent and multiagent chemotherapy regimens ${ }^{3-5}$ and more recently with other systemic therapies such as immunotherapy and biologic therapies. ${ }^{6,7}$

At this institution an $\mathrm{IgG}_{1}$ murine monoclonal antibody $(\mathrm{mAb})$ designated mT84.66 was developed with a high specificity and affinity $\left(\sim 2 \times 10^{10} \mathrm{M}^{-1}\right)$ for carcinoembryonic antigen (CEA). ${ }^{8}$ The antibody has been conjugated to diethylenetriaminepentaacetic acid (DTPA) or tetraazacyclodocecane-tetraacetic acid (DOTA) and radiolabeled for clinical studies. Indium-111 ( ${ }^{111}$ In)-DTPAmT84.66 demonstrated successful targeting and imaging of colorectal cancer. ${ }^{9}$ The antibody was engineered to reduce immunogenicity by humanizing the nonantigen binding constant domains of the antibody creating a human/murine chimeric version, designated as chimeric T84.66 (cT84.66) mAb. A pretherapy imaging trial evaluating ${ }^{111}$ In-DTPA-cT84.66 demonstrated tumor targeting and minimal toxicity of the antibody. ${ }^{10}$ A subsequent phase I trial of yttrium-90 $\left({ }^{90} \mathrm{Y}\right)$ DTPA-cT84.66 established a maximum tolerated dose (MTD) of $16.6 \mathrm{mCi} / \mathrm{m}^{2}$ with myelosuppression being dose limiting. ${ }^{11}$ Thirteen of $22(59 \%)$ developed a human antichimeric antibody (HACA) response, limiting the number of additional cycles of RIT. In a subsequent phase I trial with ${ }^{90}$ Y-DOTAcT84.66, ${ }^{12}$ dose-limiting hematologic toxicity was observed and an MTD of $13.4 \mathrm{mCi} / \mathrm{m}^{2}$ was established. Eight of 13 patients $(62 \%)$ developed a HACA response preventing subsequent therapy cycles in 4 patients. Subsequent phase I trials demonstrated the feasibility of combining radiosensitizing single agent chemotherapy with ${ }^{90} \mathrm{Y}$-DOTA-cT84.66 RIT at $16.6 \mathrm{mCi} / \mathrm{m}^{2}$ with $1000 \mathrm{mg} /\left(\mathrm{m}^{2} \cdot \mathrm{d}\right) 5$-fluorouracil $(5-\mathrm{FU})$ given as a 5-d continuous infusion ${ }^{3}$ or with gemcitabine at $150 \mathrm{mg} / \mathrm{m}^{2}$ given on days 1 and 3 after RIT. ${ }^{4}$

To further reduce immunogenicity of cT84.66 mAb a humanized version was engineered and designated hT4.66-M5A (M5A) mAb. A phase I trial of ${ }^{90}$ Y-DOTAM5A anti-CEA RIT was then carried out to evaluate the immunogenicity of M5A and to determine the MTD and associated dose-limiting toxicities (DLTs), initially in combination with gemcitabine and then as a single agent.

\section{Materials and Methods}

\section{Antibody production and conjugation}

The hT84.66-M5A (M5A) mAb is a humanized $\mathrm{IgG}_{1}$ $\mathrm{mAb}$ derived from the murineT84.66 mAb by CDR grafting based on structure design. ${ }^{13}$ The M5A mAb was expressed, purified, and conjugated to the macrocyclic chelate 1,4,7,10-tetraazacylcodecane- $\mathrm{N}, \mathrm{N}^{\prime}, \mathrm{N}, \mathrm{N}^{\prime \prime} \mathrm{N}^{\prime \prime}$-tetraacetic acid (DOTA) at the City of Hope Center for Biologics and Genetics under cGMP regulations in accordance with FDA Investigational New Drug application. The antibody was labeled with ${ }^{111} \mathrm{In}$ and ${ }^{90} \mathrm{Y}$ to form the imaging or therapeutic agent, respectively. ${ }^{12}$

\section{Clinical trial design}

The primary objective of this trial was to establish the MTD of ${ }^{90}$ Y-DOTA-M5A humanized anti-CEA antibody and to characterize associated toxicities initially in combination with gemcitabine and then as a single agent. Patients with advanced chemotherapy refractory metastatic CEA producing malignancies for which no standard therapies were available were eligible. Patients were required to have at least one measurable site of disease. Patients with untreated brain metastases were excluded. CEA expression was documented by either an elevated serum CEA level or positive immunohistochemistry. Other eligibility criteria included measurable disease; Karnofsky performance status of $\geq 60 \%$; no therapy in the 4 weeks before study entry; adequate bone marrow function as defined by WBC $\geq 4000$ / $\mu \mathrm{L}$, absolute neutrophil count $\geq 1500 / \mu \mathrm{L}$, and platelet count $\geq 125,000 / \mu \mathrm{L}$; creatinine $<1.5 \mathrm{mg} / \mathrm{dL}$ or creatinine clearance $>60 \mathrm{cc} / \mathrm{min}$; bilirubin $\leq 1.5 \mathrm{mg} / \mathrm{dL}$; and liver transaminases no greater than two times the upper limit of normal. This study was reviewed and approved by the City of Hope Institutional Review Board (IRB) and all patients were consented to the study.

Before treatment, each patient received $5 \mathrm{mCi} / 5 \mathrm{mg}{ }^{111} \mathrm{In}$ DOTA-M5A infused intravenously for $25 \mathrm{~min}$. This was followed by planar $\gamma$ camera imaging at $1-3 \mathrm{~h}, 1 \mathrm{~d}, 2 \mathrm{~d}, 3-5$ d, and 6-7 d postinfusion. Single photon emission computed tomography imaging was also performed at 2 and $3-5 \mathrm{~d}$ postinfusion. Serial blood samples were collected at $\sim 0,1$, and 4-6h as well as in conjunction with the $2,3-5$, and 6-7 d scans. Round-the-clock urine collections were performed for five consecutive days beginning immediately after infusion.

Patients went on to receive the planned therapy dose of ${ }^{90}$ Y-DOTA-M5A, if antibody imaging of at least one known tumor site was observed. The therapy dose was administered no more than 2 weeks after the ${ }^{111}$ In-DOTA-M5A antibody infusion. The infusion of ${ }^{90}$ Y-DOTA-M5A RIT included 5 $\mathrm{mCi}$ of ${ }^{111}$ In-DOTA-M5A. The total amount of antibody protein infused was kept constant at $5 \mathrm{mg}$. As in prior studies, to minimize any free ${ }^{90} \mathrm{Y}$ targeting to bone (thus increasing bone marrow toxicity) patients received a Ca-DTPA infusions after ${ }^{90}$ Y-DOTA-M5A administration at a dose of $250 \mathrm{mg} /\left(\mathrm{m}^{2}\right.$ per $24 \mathrm{~h}$ ) for $3 \mathrm{~d}$ given in equally divided doses every $12 \mathrm{~h}$. Serial $\gamma$ camera scans, blood sampling, and urine collections were performed at the same time points as for the pretherapy infusion. If no DLTs were observed and toxicities returned to grade 1 or less, a second cycle of ${ }^{90}$ Y-DOTA-M5A was delivered 6-10 weeks later. A maximum of two therapy cycles were allowed on study. Blood samples were also collected before each therapy cycle and at 3 and 6 months after RIT completion to assess for human antihuman antibody (HAHA) response using a method previously described. ${ }^{14}$ Initially, for the first two cohorts, gemcitabine was given on days 1 and 3 at $150 \mathrm{mg} / \mathrm{m}^{2}$; however, on subsequent cohorts only the single agent ${ }^{90}$ Y-DOTA-M5A was delivered.

Following a standard phase I dose escalation design, 3-6 patients were entered per dose level. The MTD was defined as the highest dose level at which $<33 \%$ of patients at that dose level experienced DLTs. DLT in a given patient was defined as any grade 3 nonhematologic toxicity or any grade 4 toxicity hematologic or nonhematologic toxicity related to the study drug. DLT was based on the first course of treatment. Toxicity was graded according to the NCI common toxicity criteria version 3.0.

Radiologic studies, including computed tomography (CT) scans, were repeated at 5-6 weeks post-therapy to assess 
tumor response. Response evaluation criteria in solid tumors were used. Response criteria were defined as follows: complete response: disappearance of all measurable and evaluable disease and no new lesions; partial response: at least a $30 \%$ decrease in the sum of the longest diameter (LD) of all measurable lesions, with no progression of evaluable disease and development of new lesions; stable disease: does not qualify for complete response, partial response, or progression; progressive disease: at least a $20 \%$ increase in the sum of LD of all measurable lesions or the appearance of one or more new lesions. Response endpoints also included overall survival (OS) and progression-free survival (PFS).

\section{Pharmacokinetic analysis and absorbed dose estimates}

Biodistributions and radiation doses for ${ }^{90}$ Y-DOTA-M5A were estimated based on the measured pharmacokinetics of ${ }^{111}$ In-DOTA-M5A, corrected for the difference in physical decay rates for the two radionuclides. Blood and urine samples were assayed for ${ }^{111}$ In activity with a $\gamma$ counter. Samples containing both ${ }^{111}$ In and ${ }^{90} \mathrm{Y}$ were counted sequentially in $\gamma$ and $\beta$ well counters. In the latter case, Cerenkov radiation was used, with quench correction, to determine the amount of ${ }^{90} \mathrm{Y}$ present. Samples were homogenized in aqueous media and bleached before counting. Standards were used to calibrate the absolute accuracy of the counting systems.

Biodistributions of ${ }^{111}$ In-DOTA-M5A were determined by planar $\gamma$ camera imaging. For features seen in both projections, ${ }^{111}$ In activity was estimated as a function of time using nonoverlapping regions of interest drawn on the geometric mean of parallel-opposed images. Otherwise, single-view images were used to estimate region of interest activity versus time. Image-derived ${ }^{111}$ In fraction of injected activity curves [FIA $(\mathrm{t})=$ total activity content/injected activity] were determined for kidneys, spleen, liver, whole body, and whole body remainder (i.e., whole body minus measured organs). Organ FIAs (which included activity in blood contents) were corrected for background counts, counts from overlying tissue (estimated from thigh muscle), and photon attenuation in tissue. Corrections for attenuation and overlying activity employed measurements of body and organ thicknesses ob- tained from patients' CT scans. Attenuation coefficients were derived from experiments that determined $\gamma$ camera counting efficiency for a planar ${ }^{111}$ In phantom source as a function of tissue-equivalent absorber thickness. Image-derived whole body FIAs (also corrected for background counts) were evaluated assuming that no activity was excreted until after the first imaging time point. ${ }^{111}$ In whole body and liver FIAs, as well as directly measured urine FIAs and blood activity concentrations versus time, were used as inputs for a five compartment modeling analysis to estimate mutually consistent FIAs and residence times (i.e., time-integrated FIA curves) for ${ }^{90} \mathrm{Y}$ in blood, urine, liver, and whole body. ${ }^{10}$ FIAs and residence times for red marrow were estimated based on the model-derived FIAs for blood. ${ }^{15}$ Residence times for ${ }^{90} \mathrm{Y}$ DOTA-M5A in kidneys, spleen, and whole body remainder were calculated from the image-derived ${ }^{90} \mathrm{Y}$-decayed FIAs. Residence times for ${ }^{90} \mathrm{Y}$-DOTA-M5A were input to OLINDA/EXM v.1.0 ${ }^{16}$ and used in conjunction with OLINDA's male and female numerical phantoms to calculate tissue, organ, and total body absorbed doses.

\section{Results}

Eighteen patients with CEA producing malignancies were enrolled and received a pretherapy infusion on ${ }^{111}$ In-DOTAM5A. Median age was 67.5 years (49-78). Two patients with colon cancer did not go on to receive ${ }^{90} \mathrm{Y}-\mathrm{M} 5 \mathrm{~A}$ therapy. One patient failed to demonstrate tumor targeting. In a second patient ${ }^{111}$ In-DOTA-M5A imaging identified a brain lesion confirmed to be a brain metastasis on subsequent brain magnetic resonance imaging scan (Fig. 1). Of the remaining 16 patients who received therapy, 10 patients had colon cancer, 2 rectal cancer, 1 nonsmall cell lung cancer, 2 breast cancer, and 1 medullary thyroid cancer (Table 1). One of these 16 patients also had a previously unknown solitary brain metastasis seen on ${ }^{111}$ In-DOTAM5A imaging, which was treated with stereotactic radiosurgery. Subsequent restaging demonstrated control of her brain metastasis allowing her to receive therapy on trial.

A previous phase I trial demonstrated the feasibility of ${ }^{90} \mathrm{Y}$-DOTA-cT4.66 RIT at $16.6 \mathrm{mCi} / \mathrm{m}^{2}$ (day 1) combined with gemcitabine (days 1 and 3 ) reaching a gemcitabine
FIG. 1. Detection of brain metastasis by ${ }^{111}$ In-DOTA-M5A (left) confirmed on subsequent magnetic resonance imaging scan (right). ${ }^{111} \mathrm{In}$, indium-111; DOTA, tetraazacyclodocecane-tetraacetic acid.
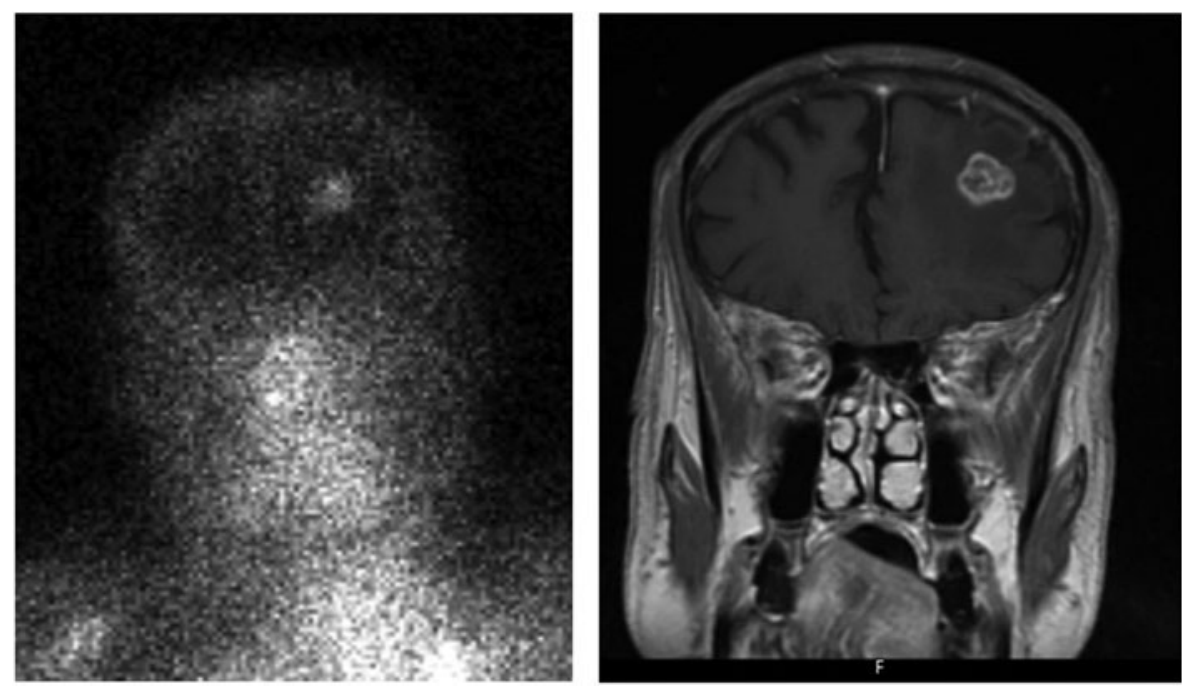
Table 1. Patient Summary Demographic Data

\begin{tabular}{lc}
\hline Patient demographics & All patients $(\mathrm{n}=18)$ \\
\hline Age (years), median (range) & $67.5(49-78)$ \\
Gender, $n(\%)$ & $8(44.4)$ \\
F & $10(55.6)$ \\
M & $12(66.7)$ \\
Diagnosis site, $n(\%)$ & $1(5.6)$ \\
Colon & $2(11.1)$ \\
Lung & $2(11.1)$ \\
Rectum & $1(5.6)$ \\
Breast & \\
Thyroid & \\
\hline
\end{tabular}

F, female; M, male.

MTD of $150 \mathrm{mg} / \mathrm{m}^{2}{ }^{4}$ Therefore, per trial design the initial cohort of 3 patients received $12 \mathrm{mCi} / \mathrm{m}^{2}{ }^{90} \mathrm{Y}$-DOTA-M5A on day 1 followed by gemcitabine at $150 \mathrm{mg} / \mathrm{m}^{2}$ on days 1 and 3. A subsequent patient received $14 \mathrm{mCi} / \mathrm{m}^{2}{ }^{90} \mathrm{Y}$-DOTAM5A followed by gemcitabine. Three of these 4 patients ( 2 at $12 \mathrm{mCi} / \mathrm{m}^{2}$ and 1 at $14 \mathrm{mCi} / \mathrm{m}^{2}$ ) demonstrated count nadirs occurring at week one and more prolonged grade 3 thrombocytopenia and leukopenia compared with prior experience with ${ }^{90} \mathrm{Y}$-DOTA-cT84.66 alone. The trial was, therefore, amended to eliminate gemcitabine for subsequent patients. Six patients were then treated at $12 \mathrm{mCi} / \mathrm{m}^{2}{ }^{90} \mathrm{Y}$ DOTA-M5A without gemcitabine. Two of the 6 demonstrated DLTs with grade 2 leukopenia and thrombocytopenia not returning to grade 1 by 10 weeks post-RIT. For the next cohort of 6 patients the dose was reduced to $10 \mathrm{mCi} / \mathrm{m}^{2}$. None of these 6 patients experienced a DLT. Details of toxicities are described in Table 2. Table 3 displays estimated radiation doses to critical organs and total body from ${ }^{90} \mathrm{Y}$-DOTA-M5A and compared with those previously seen with ${ }^{90}$ Y-DOTA-cT84.66. ${ }^{12}$ No differences were observed between the two agents.

In summary, 16 patients received ${ }^{90} \mathrm{Y}$-DOTA-M5A therapy, 6 at $10 \mathrm{mCi} / \mathrm{m}^{2}, 9$ at $12 \mathrm{mCi} / \mathrm{m}^{2}$, and 1 at $14 \mathrm{mCi} / \mathrm{m}^{2}$. Total administered activity ranged from 14.4 to $26.5 \mathrm{mCi}$ of ${ }^{90} \mathrm{Y}$ (Table 4). Six patients received two cycles of therapy. Seven patients completed treatment, whereas 5 patients were taken off protocol due to disease progression. Two patients $(12.5 \%)$ were taken off protocol due to development of HAHA, which is less $(p<0.01$, Fisher's exact test) than the $60 \%$ (21/35) patients with HACA previously treated with ${ }^{90}$ Y-DOTA-cT4.66 RIT. ${ }^{11,12}$ One patient declined a second cycle (Table 5).

Although no objective responses were observed in this patient population with chemotherapy refractory disease, stable disease at 3 months was seen in 10 of 16 patients

TABLE 2. ToXicities

\begin{tabular}{lccc}
\hline Treatment level & $\begin{array}{c}10 \\
\mathrm{mCi} / \mathrm{m}^{2}\end{array}$ & $\begin{array}{c}12 \\
\mathrm{mCi} / \mathrm{m}^{2}\end{array}$ & $\begin{array}{c}14 \\
\mathrm{mCi} / \mathrm{m}^{2}\end{array}$ \\
\hline $\begin{array}{l}\text { Grade } 3 \text { prolonged cytopenia } \\
\text { Grade 2 counts not } \leq \mathrm{G} 1\end{array}$ & 0 & $2^{\mathrm{a}}$ & $1^{\mathrm{a}}$ \\
$\begin{array}{l}\text { by 10 weeks } \\
\text { Grade 2 urticaria }\end{array}$ & 0 & 2 & 0 \\
\hline
\end{tabular}

${ }^{\mathrm{a}}$ With gemcitabine $150 \mathrm{mg} / \mathrm{m}^{2}$ on days 1 and 3 .
Table 3. Estimated Radiation Yttrium-90 Doses to Normal Organs (CGy/MCI)

\begin{tabular}{lcc}
\hline & $\begin{array}{c}{ }^{90} \text { Y-DOTA-M5A } \\
(\text { current study) } \\
\text { Target organ } \\
(\mathrm{n}=16)\end{array}$ & $\begin{array}{c}90 \text { Y-DOTA-cT84.66 } \\
\text { Yean }(\text { range })^{\mathrm{a}} \\
(n=15)\end{array}$ \\
\hline Kidneys & $10.6(3.7-20.0)$ & $5.4(0.7-14.4)$ \\
Liver & $31.8(12.9-55.6)$ & $35.9(19.5-54.7)$ \\
Lungs & $1.2(0.8-1.9)$ & $1.1(0.7-1.5)$ \\
Red marrow & $2.4(1.0-4.8)$ & $2.2(0.9-3.8)$ \\
Total body & $2.2(1.9-2.7)$ & $2.1(1.8-2.6)$ \\
\hline
\end{tabular}

${ }^{\mathrm{a}}$ Data are means and ranges of intrapatient average doses determined from pre- and cotherapy administrations of ${ }^{111}$ In-DOTA-M5A.

${ }^{90} \mathrm{Y}$, yttrium-90; ${ }^{111}$ In, indium-111; DOTA, tetraazacyclodocecanetetraacetic acid.

(62.5\%), including 5 of 6 treated on single agent ${ }^{90}$ Y-DOTAM5A at the MTD. In addition, 2 patients demonstrated a reduction of serum CEA levels back to normal, 1 who received two cycles of RIT at $10 \mathrm{mCi} / \mathrm{m}^{2}$ demonstrated an $88 \%$ decrease in CEA, and another who received two cycles at $12 \mathrm{mCi} / \mathrm{m}^{2}$ and gemcitabine demonstrated a 64\% decrease in CEA. Two other patients had $21 \%$ and $12 \%$ decreases in CEA. The median OS was 8.7 months (95\% confidence interval [CI]: 5.8-29.2) and the median PFS was 2.8 months (95\% CI: 1.2-5.8). Three patients who received ${ }^{90}$ Y-DOTA-M5A as a single agent had prolonged survival of 29.2, 39.7, and $>53.1$ months.

\section{Discussion}

Radiolabeled antibodies have demonstrated potential for diagnostic and therapeutic purposes. The purpose of this first in human phase I trial was to evaluate the safety and to determine the MTD and associated DLTs of ${ }^{90} \mathrm{Y}$-DOTAM5A anti-CEA RIT initially with gemcitabine and subsequently as a single agent. One limitation of earlier mAbs used for RIT was the development of human anti-marine antibody (HAMA) or HACA responses, limiting multiple administrations. Murine mAbs typically result in HAMA responses in $80 \%-100 \%$ of patients after single administration. ${ }^{17-20}$ Chimeric mAbs reduce immunogenicity with HACA responses ranging from $40 \%$ to $80 \% .^{21-24}$ With the cT84.66 mAb 13 of 22 patients (59\%) developed HACA in a phase I trial of ${ }^{90} \mathrm{Y}$-DTPA-cT84.66. ${ }^{11}$ In another phase I trial of ${ }^{90} \mathrm{Y}$-DOTA-cT84.66, 8 of 13 patients $(62 \%)$ developed HACA. $^{12}$ This was the main impetus for development the humanized M5A antibody, which demonstrated significantly lower immunogenicity with only 2 of 16 patients (12.5\%) developing HAHA.

Table 4. Summary of Yttrium-90 M5A Dose ESCALATION AND ADMINISTERED ACTIVITY

\begin{tabular}{lcc}
\hline Dose level & $\begin{array}{c}\text { No. of } \\
\text { patients }\end{array}$ & $\begin{array}{c}\text { Actual dose } \\
\text { Mean (mCi) } \\
\text { (range) }\end{array}$ \\
\hline $10 \mathrm{mCi} / \mathrm{m}^{2}$ & 6 & $18.6(14.4-22.7)$ \\
$12 \mathrm{mCi} / \mathrm{m}^{2}$ & 9 (3 with gem) & $21.1(18.6-26.5)$ \\
$14 \mathrm{mCi} / \mathrm{m}^{2}$ & 1 (with gem) & 21.7 \\
\hline
\end{tabular}

gem, gemcitabine. 
Table 5. Patient Outcomes

\begin{tabular}{lc}
\hline Treatment and outcomes & All patients $(\mathrm{n}=16)$ \\
\hline Dose level, $n(\%)$, mean dose in $\mathrm{mCi}(\mathrm{range})$ \\
$10 \mathrm{mCi} / \mathrm{m}^{2}$ & $6(37.5), 18.6(14.4-22.7)$ \\
$12 \mathrm{mCi} / \mathrm{m}^{2}$ & $9(56.3), 21.1(18.6-26.50$ \\
$14 \mathrm{mCi} / \mathrm{m}^{2}$ & $1(6.3), 21.7$ \\
No. of RIT cycles, $n(\%)$ & \\
1 only & $6(62.5)$ \\
2 & \\
HAHA, $n(\%)$ & $2(12.5)$ \\
Positive & $14(87.5)$ \\
Negative & $10(62.5)$ \\
Best response, $n(\%)$ & $6(37.5)$ \\
Stable & \\
Progression & $7(43.8)$ \\
Reason off RIT, $n(\%)$ & $1(6.3)$ \\
Protocol completion & $2(12.5)$ \\
Prolonged cytopenia & $5(31.3)$ \\
HAHA & $1(6.3)$ \\
Progressive disease & 8.7 months $(5.8-29.2)$ \\
Patient choice & \\
OS median OS (months) & \\
(95\% CI) & \\
PFS from treatment start date & 2.8 months $(1.2-5.8)$ \\
Median PFS (months) & \\
(95\% CI) & \\
\hline
\end{tabular}

CI, confidence interval; HAHA, human antihuman antibody; OS, overall survival; PFS, progression-free survival; RIT, radioimmunotherapy.

As with most RIT agents DLT was leukopenia and thrombocytopenia. An MTD of $10 \mathrm{mCi} / \mathrm{m}^{2}$ for ${ }^{90} \mathrm{Y}$-DOTAM5A as a single agent was determined. This is lower than the MTD of $16.6 \mathrm{mCi} / \mathrm{m}^{2}$ reached for ${ }^{90} \mathrm{Y}$-DTPA-cT84.66 ${ }^{11}$ and $13.4 \mathrm{mCi} / \mathrm{m}^{2}$ for ${ }^{90} \mathrm{Y}$-DOTA-cT84.66. ${ }^{12}$ This is not related to significant differences in absorbed radiation doses to red marrow, total body, or other critical organs since doses were similar as in the previous studies. The difference most likely can be explained by the fact that patients in earlier studies primarily had failed only 5-FU before study entry, whereas patients in the current study had progressed on not only 5-FU, but newer chemotherapy agents such as oxaliplatin and irinotecan, and were, therefore, more heavily pretreated with less marrow reserve.

Although this study was primarily to characterize immunogenicity, toxicities, and define an MTD, there was evidence of antitumor effects in this heavily pretreated population with advanced disease. Ten of 16 patients $(62.5 \%)$ demonstrated stable disease. In addition, 2 patients demonstrated an $88 \%$ and $64 \%$ decrease in CEA to within normal limits. These results are similar to other RIT agents directed against stolid tumors and reflect tumor doses achievable by RIT alone. Median and mean doses have been reported to be $\sim 10-20$ Gy. Although these doses will have limited impact on bulky macroscopic disease, they can potentially result in clinically important antitumor effects, particularly in subclinical or microscopic disease settings. Future studies should, therefore, evaluate RIT as part of a combined modality approach in combination with established systemic chemotherapy, biologic therapy, or immunotherapy. Radiolabeling M5A with other radionuclides, such as lutetium-177 or $\alpha$ emitters, may have greater therapeutic potential and should also be evaluated in preclinical models and in clinical trials in the future.

This study also demonstrated the imaging potential of

${ }^{111}$ In-DOTA-M5A with only 1 out of 18 patients not demonstrating tumor targeting. ${ }^{111}$ In-DOTA-M5A also detected a previously unknown brain metastasis in 2 patients. Radiolabeling of M5A with a PET emitting radionuclide has the potential to further improve the imaging potential of this antibody.

In conclusion, this phase I study demonstrates that M5A, a humanized version of the cT84.66 anti-CEA mAb, has reduced immunogenicity compared with cT84.66, promising tumor imaging capabilities, and when labeled with ${ }^{90} \mathrm{Y}$ is associated with some therapeutic effect in heavily pretreated patients with advanced refractory disease.

\section{Disclosure Statement}

No competing financial interests exist.

\section{Authorship Confirmation Statement}

D.A. performed data compilation, data analysis, and article preparation; D.Y. did scan interpretation; J.S. did data collection and data compilation; P.H.F. did the trial design, statistical analysis, and article preparation; K.P. did antibody radiolabeling; J.B. did radiation dose estimates; P.Y., A.W., D.C., and J.S. were in charge of antibody design, antibody production, antibody conjugation, and IND submission; Y.C., D.L., and M.C. performed patient accrual and trial execution; J.Y.C.W. was responsible for trial design, IND submission, trial implementation and execution, patient accrual, data compilation, data analysis, and article preparation. All coauthors have reviewed and approved the article before submission.

\section{Funding Information}

This study was supported by NIH PO1 43904 and NIH Cancer Center Core Grant 33572.

\section{References}

1. Wiseman GA, White CA, Stabin M, et al. Phase I/II ${ }^{90} \mathrm{Y}-$ Zevalin (yttrium-90 ibritumomab tiuxetan, IDEC-Y2B8) radioimmunotherapy dosimetry results in relapsed or refractory non-Hodgkin's lymphoma. Eur J Nucl Med 2000; 27:766.

2. Horning SJ, Younes A, Jain V, et al. Efficacy and safety of tositumomab and Iodine-131 tositumomab (Bexxar) in Bcell lymphoma progressive after rituximab. J Clin Oncol 2005;23:712.

3. Wong JYC, Shibata S, Williams LE, et al. A phase I trial of Y-90-anti-carcinoembryonic antigen chimeric T84.66 radioimmunotherapy with 5-fluorouracil in patients with metastatic colorectal cancer. Clin Cancer Res 2003;9:5842.

4. Shibata S, Raubitschek A, Leong L, et al. A Phase I study of a combination of yttrium-90 labeled anti-CEA antibody and gemcitabine in patients with CEA producing advanced malignancies. Clin Cancer Res 2009;15:2935.

5. Picozzi VJ, Ramanathan RK, Lowery MA, et al. 90Yclivatuzumab tetraxetan with or without low-dose gemcitabine: A phase Ib study in patients with metastatic pancreatic cancer after two or more prior therapies. Eur $\mathbf{J}$ Cancer 2015;51:1857. 
6. Bodet-Milin C, Salaun P-Y, Paris F, et al. Toxicity and efficacy of combined radioimmunotherapy and bevacizumab (avastin) in mouse model of medullary thyroid carcinoma [abstr]. Cancer Biother Radiopharm 2008;23:517.

7. Mach J-P. Recombinant monoclonal antibodies, from tumor targeting to cancer immunotherapy: A critical overview. Mol Biol 2017;51:1024.

8. Neumaier M, Shively L, Chen FS, et al. Cloning of the genes for T84.66, an antibody that has a high specificity and affinity for carcinoembryonic antigen, and expression of chimeric human/mouse T84.66 genes in myeloma and Chinese hamster ovary cells. Cancer Res 1990;50:2128.

9. Beatty JD, Duda RB, Williams LE. Pre-operative imaging of colorectal carcinoma with 111-In-labled anticarcinoembryonic antigen monoclonal antibody. Cancer Res 1986;46:6494.

10. Wong JYC, Thomas GE, Yamauchi DM, et al. Clinical evaluation of Indium-111-labeled chimeric anti-CEA monoclonal antibody. J Nucl Med 1997;38:1951.

11. Wong JYC, Chu DZ, Yamauchi DM, et al. A Phase I radioimmunotherapy trials evaluating Y-90 labeled antiCEA chimeric T84.66 in patients with metastatic CEAproducing malignancies. Clin Cancer Res 2000;6:3855.

12. Wong JYC, Chu DZ, Williams LE, et al. A Phase I trial of ${ }^{90}$ Y-DOTA-Anti-CEA chimeric T84.66 (cT84.66) radioimmunotherapy in patients with metastatic CEAproducing malignancies. Cancer Biother Radiopharm 2006;21:88.

13. Yazaki PJ, Sherman MA, Shively JE, et al. Humanization of the anti-CEA T84.66 antibody based on crystal structure data. Protein Eng Des Sel 2004;17:481.

14. Wong JY, Thomas GE, Yamauchi D, et al. Clinical evaluation of indium-111-labeled chimeric anti-CEA monoclonal antibody. J Nucl Med 1997;38:1951.
15. Siegel JA, Wessels BW, Watson EE, et al. Bone marrow dosimetry and toxicity for radioimmunotherapy. Antibody Immunoconj Radiopharm 1990;3:213.

16. Stabin MG, Sparks RB, Crowe E. OLINDA/EXM: The secondgeneration personal computer software for internal dose assessment in nuclear medicine. J Nucl Med 2005;46:1023.

17. Breitz HB, Weiden PL, Vanderheyden JL, et al. Clinical experience with rhenium-186-labeled monoclonal antibodies for radioimmunotherapy: Results of phase I trials. J Nucl Med 1992;33:1099.

18. Meredith RF, Bueschen AJ, Khazaeli MB, et al. Treatment of metastatic prostate carcinoma with radiolabeled antibody CC49. J Nucl Med 1994;35:1017.

19. DeNardo SJ, Kramer EL, O'Donnell RT, et al. Radioimmunotherapy for breast cancer using indium-111/ yttrium-90 BrE- 3: Results of a phase I clinical trial. J Nucl Med 1997;38:1180.

20. Divgi CR, Bander NH, Scott AM, et al. Phase I/II radioimmunotherapy trial with iodine-131-labeled monoclonal antibody G250 in metastatic renal cell carcinoma. Clin Cancer Res 1998;4:2729.

21. Meredith RF, Khazaeli MB, Plott WE, et al. Phase I trial of iodine-131-chimeric B72.3 (Human IgG4) in metastatic colorectal cancer. J Nucl Med 1992;33:23.

22. Meredith RF, Khazaeli MB, Plott WE, et al. Initial clinical evaluation of iodine-125-labeled chimeric 17-1A for metastatic colon cancer. J Nucl Med 1995;36:2229.

23. DeNardo SJ, O'Grady LF, Richman CM, et al. Radioimmunotherapy for advanced breast cancer using I-131ChL6 antibody. Anticancer Res 1997;17:1745.

24. Colnot DR, Quak JJ, Roos JC, et al. Phase I therapy study of ${ }^{186}$ re-labeled chimeric monoclonal antibody U36 in patients with squamous cell carcinoma of the head and neck. J Nucl Med 2000;41:1999. 